\title{
Detection of gait perturbations based on proprioceptive information. Application to Limit Cycle Walkers
}

\author{
J.A. Gallego ${ }^{\mathrm{a}, *}$, A. Forner-Cordero ${ }^{\mathrm{b}}$, J.C. Moreno ${ }^{\mathrm{a}}$, E.A. Turowska ${ }^{\mathrm{a}}$ and J.L. Pons ${ }^{\mathrm{a}}$ \\ ${ }^{a}$ Bioengineering Group, Consejo Superior de Investigaciones Científicas, CSIC, Arganda del Rey, \\ Madrid, Spain \\ ${ }^{\mathrm{b}}$ Biomechatronics Laboratory, Mechatronics and Mechanical Systems Department, University of Sao Paulo, \\ Sao Paulo, Brazil
}

\begin{abstract}
Walking on irregular surfaces and in the presence of unexpected events is a challenging problem for bipedal machines. Up to date, their ability to cope with gait disturbances is far less successful than humans': Neither trajectory controlled robots, nor dynamic walking machines (Limit Cycle Walkers) are able to handle them satisfactorily. On the contrary, humans reject gait perturbations naturally and efficiently relying on their sensory organs that, if needed, elicit a recovery action. A similar approach may be envisioned for bipedal robots and exoskeletons: An algorithm continuously observes the state of the walker and, if an unexpected event happens, triggers an adequate reaction. This paper presents a monitoring algorithm that provides immediate detection of any type of perturbation based solely on a phase representation of the normal walking of the robot. The proposed method was evaluated in a Limit Cycle Walker prototype that suffered push and trip perturbations at different moments of the gait cycle, providing $100 \%$ successful detections for the current experimental apparatus and adequately tuned parameters, with no false positives when the robot is walking unperturbed.
\end{abstract}

Keywords: Perturbation detection, dynamic stability, bipedal robots, Limit Cycle Walking, basin of attraction

\section{Introduction}

Research on gait synthesis constitutes a constructive manner to increase our understanding of the principles underlying human walking. This knowledge is useful in two types of applications: human-centered applications, and robotics-centered applications. The former include the design of improved rehabilitation devices such as robotic exoskeletons or prostheses [3, 23], whereas the latter will translate into the development of humanoid robotic companions and caretakers,

\footnotetext{
*Corresponding author. E-mail: gallego@iai.csic.es.
}

entertainment robots, and social interaction robots in general $[16,27]$.

Unfortunately, taking these systems from the laboratories to our daily context is yet not entirely possible, because our real world is full of non expected events that may lead the walker to a fall, e.g. obstacles, ground irregularities, slope changes, and collisions with other robots or humans. Therefore, recovery from gait perturbations and balance control emerge as major topics in bipedal walking.

Concerning to the rejection of perturbations, humans cope with this problem in a quite successful manner choosing among a reduced repertoire of strategies, 
which are adapted to the specific context [10, 28]. When a perturbation takes place, multiple sensory receptors trigger a certain reaction, trying to avoid a fall [8]. In fact, stumbling reactions or biological recovery motions to keep the human body stable during walking, are -partially- modulated by peripheral afferent signals [7]. Thus, kinesthetic information needs to be fedback in order to update the central motor program, which can be regarded as a modulated closed system [20]. Considering this, it seems interesting to investigate a similar solution for robotic walkers and exoskeletons: An algorithm continuously monitors the state of the biped and, if necessary, triggers a recovery reaction. This is the final objective of EU project ESBiRRo (IST-61045301-STP), which aims at developing biomimetic recovery reactions for gait control that will be implemented into an autonomous biped robot and a robotic exoskeleton. This paper focuses on a general method for detection of any type of external gait perturbation in robotic walkers.

It is hypothesized that the human cerebellum generates a series of forward and inverse models, which represent the normal behavior of the motor system in response to ongoing motor commands, and the neural command required to generate a given trajectory respectively [32]. Regarding to forward representations, different structures and/or functions are attributed to them, such as output prediction, state estimation or distal teaching [18]. Functional brain imaging studies signal that the cerebellum is involved in signalling the discrepancy between the predicted and actual sensory consequences of movement [5]. Moreover, in a recent work [1], it is hypothesized that a nominal forward internal model combined with probabilistic error monitoring is employed by the Central Nervous System to detect a loss of balance and precedes any observable compensatory response.

On the other hand, gait synthesis as understood in the framework of Limit Cycle Walking, relies on keeping in the neighborhood of the limit cycle prescribed by the robot state during a stride. Indeed, it has been proven that optimization of the system dynamics makes exponentially stable, efficient, and natural gait emerge [31], and that simple torque control provides the biped with the ability to cope with varying walking speeds, ground slopes, and push perturbations [6]. This happens because the limit cycle prescribed by a system is enveloped by a surface known as basin of attraction, which contains all the states that bring the system "naturally" back to its limit cycle. However, the basin of attraction is not analytically computable for systems with such a large number of state variables as a walking robot [29]. According to this, the problem of detecting whether the walker is undergoing or not a perturbation that will lead to a fall equals to estimating whether it is inside or outside the basin of attraction.

On the basis of these two ideas, we propose a method for instability detection in Limit Cycle Walkers, which consists in estimating the deviation between the actual and expected robot state as provided by sensory signals, to subsequently compare it with a linear, probabilistic approach to the basin of attraction.

Regarding to the state of the art, research on perturbation detection in biped robots has focused on tailor made techniques, which detect a well defined perturbation in order to implement some reflex, recovery strategy, or balance control mechanism to avoid a fall. In [21], a method to detect a push in the trunk by observing the upper body acceleration in the anteroposterior direction is proposed. In a more recent work [24], they implement ankle control to permit a small walking robot adapting to both continuous perturbations (slope changes, addition of mass) and pushes. The latter are simply detected with a force sensor attached at the back of the robot.

Looking at more general detection and classifications paradigms, an algorithm to predict the fall of a prototype due to moderate ground irregularities is proposed in [17]. This method relies on monitoring the whole state of the robot and is validated with a limit cycle prototype, providing successful detection in the last heel strike before the fall. A method for instability detection during omnidirectional walking to trigger a reflex mechanism is proposed in [26]. Perturbations are classified in one of two groups according to their strength, and a different reflex is executed based on this identification. The method is validated with a real prototype that stumbles over a wall. In [14] a probability based algorithm is employed to classify the robot state in viable (i.e. keep walking), perturbed (a reflex mechanism may avoid the fall) or unavoidably leading to a fall. A similar approach is presented in [22] where the mean deviation from normal walking during a whole step is employed to activate a shock-reducing motion if a fall is foreseen.

This paper is organized as follows: first the method for detection of perturbations in robotic walkers is described. Next, Section 3 presents an example of application in the real prototype, whereas Section 4 includes the discussion and an outline of future 
research. Finally, some conclusions summarizing the main results are provided.

\section{Detection of perturbations based on the Nearest Neighbor Gait Index}

This section describes a novel algorithm for perturbation detection in robotic walkers. It is based on a normal walking pattern, allowing for real-time implementation and negligible detection delay.

The algorithm is executed in two steps, Fig. 1. First, it calculates the state in a normal walking pattern (the Reference Limit Cycle, RLC) that best represents the real state of the robot (what we call the Nearest Neighbor Gait Index, NNGI), and afterwards it computes the (weighed) deviation between this expected state and the actual state of the walker. The weighed deviation (D-statistic) provides an experimental approximation to whether the robot is inside or outside the basin of attraction based on a threshold.

Before running the algorithm, the normal walking of the robot, the Reference Limit Cycle, must be defined offline. Afterwards, during its execution, the first part of the algorithm, the application of the NNGI is subdivided in two steps: 1) the selection of a subset of possible states in the RLC, the Ensemble of Candidate Neighbors (ECN), in order to save computational burden, and 2) the search of the point within the aforementioned subset that represents best the current state of the walker, the Selection of the Nearest Neighbor (NN), Fig. 1. As said, during the second step, the algorithm computes the weighed distance between the $\mathrm{NN}$ and the current state of the walker (Section 2.2). Comparing it with a threshold corresponds to a linear approach to being inside or outside the basin of attraction. The complete procedure is described in detail in the next paragraphs.

\subsection{The Nearest Neighbor Gait Index (NNGI)}

The NNGI looks for the state in the normal walking pattern, the RLC, that matches best the current state of the robot.

\subsubsection{Definition of the Reference Limit Cycle (RLC)}

The RLC provides the normal walking of the biped. It is obtained off-line by averaging a series of stable runs on a surface with small disturbances. The disturbances should be large enough to cause variation between strides (cycles) without making the robot collapse. Notice that an analytical frontier between small and large perturbations cannot be established, because the former are those that do not make the walker collapse whereas the latter do, and this would

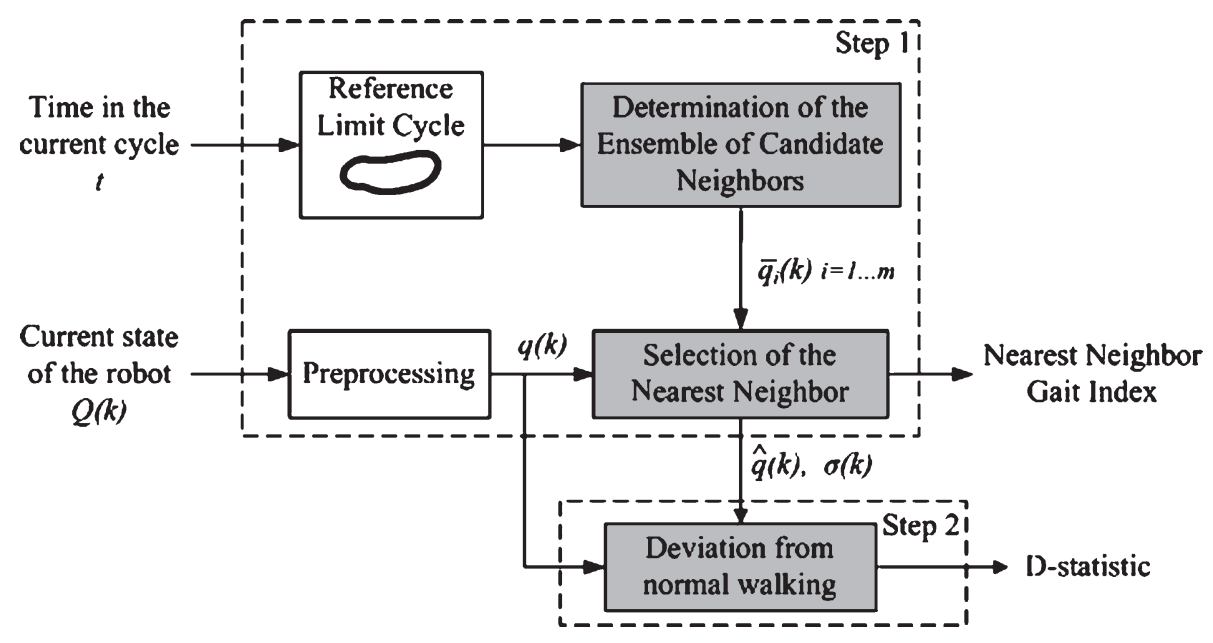

Fig. 1. Block diagram summarizing the proposed method. First, the current state of the walker $Q(k)$ is processed to obtain $q(k)$. Afterwards, based on timing information, an Ensemble of Candidate Neighbors (ECN) is selected. The ECN is a subset of the Reference Limit Cycle that represents the normal walking of the robot. Next, a Nearest Neighbor search to find the state in the ECN that matches best the current state of the robot, $\hat{q}(k)$, is performed. Finally, the deviation between the expected and current state of the walker is calculated, the D-statistic. This constitutes, together with the NNGI, the output of the algorithm. 
require computing analytically the basin of attraction, which is, as stated above not possible for a Limit Cycle Walker. Hence, we consider a perturbation to be small if it causes no (even visually) remarkable deviation from the normal gait pattern on flat terrain. In practice, small perturbations during calculation of the RLC are applied by making the biped walk on a carpeted floor, which light irregularities may be assimilated to white noise.

Because of the underactuated nature of Limit Cycle Walking, inter-stride variations in both duration and joint trajectories (and velocities) take place [11]. Therefore, to obtain the RLC the first step is to scale joint angles and angular rates to stride percentage. Next, to take the range of each joint into account and to avoid problems related to units, phase variables (joint angles and angular rates) $Q_{j}(k), j=1, \ldots, 2 n$ are divided by their respective maxima, $\max \left(\left|Q_{j}\right|\right)$, $j=1, \ldots, 2 n,(1)$. Like this, RLC variables at every instant $k$ vary within $[-1,1]$. It must be noted that the resultant scaled phase space is unbounded, i.e. any variable can be outside the $[-1,1]$ interval, for example, when the robot suffers a perturbation.

$$
q_{j}(k)=\frac{Q_{j}(k)}{\max \left(\left|Q_{j}\right|\right)}
$$

Once phase variables have been averaged to percentage and scaled, the $2 n$-dimensional RLC is obtained as the mean trajectory $\bar{q}$ of each variable for the $p$ recorded strides, (2). The standard deviation $\sigma$ of each variable at each point of the RLC is also calculated, (3), because it will be used in the measure of deviation. Afterwards $\bar{q}_{j}(k)$ and $\sigma_{j}(k)$ are resized to a number of samples that corresponds to the average duration of a stride, $r$. Note that the mean stride duration will provide an estimate of the state in the RLC that corresponds to the current state of the walker as will be discussed below.

$$
\begin{gathered}
\bar{q}_{j}(k)=\sum_{j=1}^{p} \frac{q_{j}(k)}{p}, \quad k=1, \ldots, 100 \\
\sigma_{j}(k)=\left(\frac{1}{p-1} \sum_{j=1}^{p}\left(q_{j}(k)-\bar{q}_{j}(k)\right)^{2}\right)^{1 / 2}, k=1, \ldots, 100
\end{gathered}
$$

\subsubsection{Determination of the Ensemble of Candidate Neighbors (ECN)}

Limit Cycle Walking is a nominally periodic sequence of strides, which means that in the absence of (large) perturbations each stride is almost an exact mapping of the previous one [11]. Therefore at time $t$ in step number $s$, the walker will be in a state very close to the the one it was in at time $t$ during step $s-1$. Assuming this, in order to look for the state of the RLC at which the robot is at time $t$, we can select an interval of $m$ points (the Ensemble of Candidate Neighbors, ECN) around the expected state at time $t$ : If the robot has not suffered a disturbance its state will be within this interval. This is illustrated in Fig. 2: the walker is in the state $q(k)$, and the algorithm expects it to be in the plotted interval based solely on time information. However, if the walker has undergone a perturbation, it will deviate very quickly from its expected state. The convergence time of the NNGI is equal to the number of samples of the RLC, $r$, divided by the length of the ECN, $m$. This is the first (out of two) parameter the designer has to adjust.

It must be noted that disturbances in limit cycles can be assessed in the directions tangential and transverse to the cycle, i.e. we can distinguish between those perturbations that make the system "advance" (or "go back") in the limit cycle, and those that cause a deviation in an orthogonal manifold [2]. Therefore, if the ECN is too long the algorithm will not detect perturbations that manifest in an abrupt change in the tangential direction, or it will detect them slower than with an ECN that comprises less states. Subsection 3.2 summarizes the tuning process for a Limit Cycle Walking prototype.

\subsubsection{Selection of the Nearest Neighbor $(N N)$}

The last part of the NNGI consists in finding the state of the RLC that matches best the current state of the walker. In order to reduce computational cost, and because during normal walking the correspondent state must be within the ECN, we perform a Nearest Neighbor (NN) search on there. The NN algorithm is a widely extended method for finding closest points in Euclidean spaces: it will provide directly the most similar state in the normal walking pattern. It has already been applied in off-line analysis of human gait [10].

The NN, $\hat{q}(k)=\hat{q}_{j}(k), j=1, \ldots, 2 n$ at sample $k$ is simply defined as the point within a metric space (the $m$ points $\bar{q}_{i}, i=1, \ldots, m$ in the ECN subset in our 


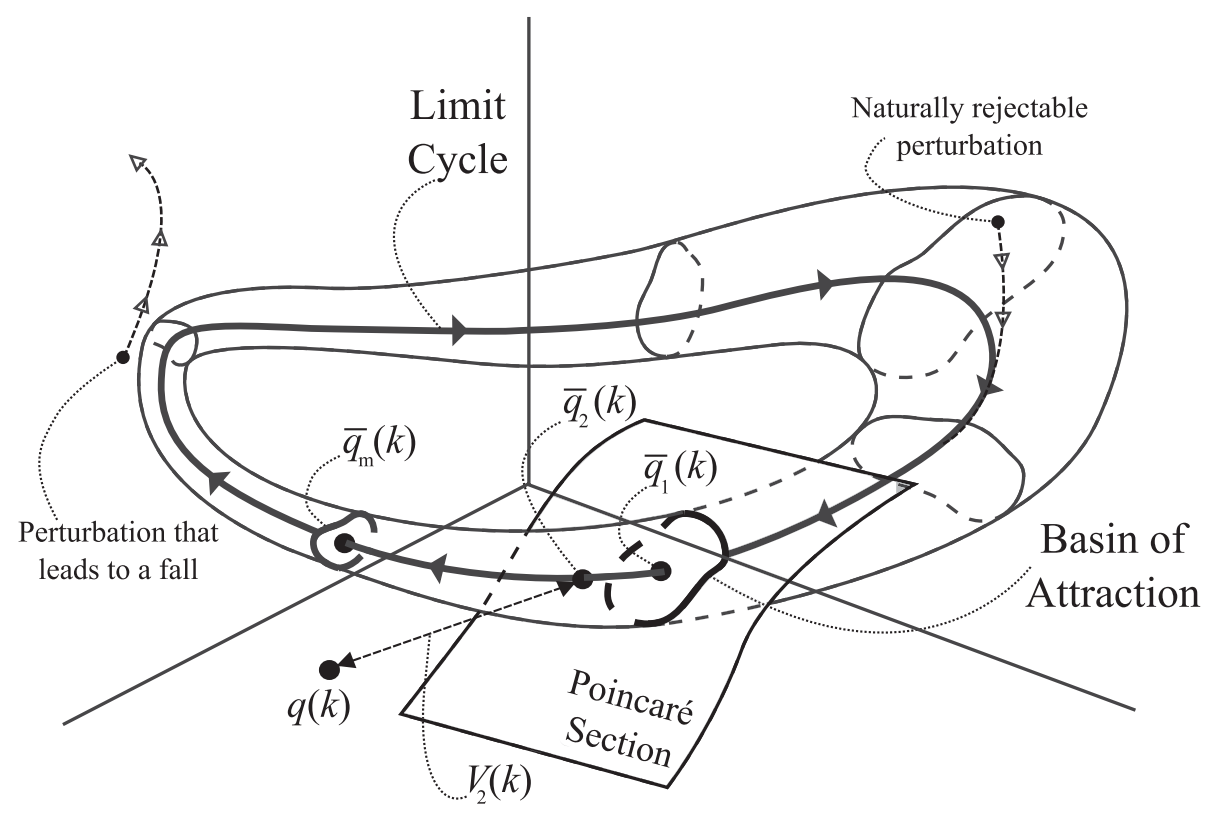

Fig. 2. 3-Dimensional abstraction of a limit cycle (solid line) together with its basin of attraction (enveloping surface). The Ensemble of Candidate Neighbors (ECN) is represented as slices in the limit cycle.

case) with the least Euclidean distance $V_{i}(k)$ to the query point, obtained after preprocessing the state of the walker with equation $1, q(k)=q_{j}(k), j=$ $1, \ldots, 2 n,(4),(5)$.

$$
\begin{gathered}
V_{i}(k)=\left(\sum_{j=1}^{2 n}\left(q_{j}(k)-\bar{q}_{j, i}(k)\right)^{2}\right)^{1 / 2}, \quad i=1, \ldots, m \\
N N(k)=\min \left\{V_{1}(k), V_{2}(k), \ldots, V_{m}(k)\right\}
\end{gathered}
$$

\subsection{Calculation of the deviation from normal walking}

Once the NNGI has selected $\hat{q}(k)$, the state in the RLC most similar to the actual state of the biped, $q(k)$, a perturbation may be detected by measuring their relative distance and estimating whether the walker is inside or outside its basin of attraction. However, the selection of an adequate measurement is not straightforward. It must be considered that a given amount of deviation does not have the same effect on dynamic stability if it takes place in different variables (i.e. processed joint angles or velocities) or even at different gait phases because the basin of attraction changes its shape and size at every point of the limit cycle. Therefore a good metric must include different weighs for each variable and time instant.

The D-statistic proposed in [17] takes both aspects into account. It consists in the squared error (between the actual and expected state of the robot) weighed by the standard deviation at that instant and variable, (6). The standard deviation quantifies the variability of a given variable during normal gait, which is related to the basin of attraction. The idea is to select a threshold for the D-statistic that separates the stable and unstable walking regions of the phase space, $D_{t h}$, mimicking the basin of attraction. This is the second parameter the designer has to tune, subsection 3.2. Note that if the designer chooses a small value for $D_{t h}$, the algorithm will detect perturbations that could be rejected naturally, but if its too large, it will fail to detect that the robot is being perturbed, or it will not provide a sufficiently fast detection.

$$
D(k)=\frac{1}{2 n-1} \sum_{j=1}^{2 n} \frac{1}{\sigma_{j}(k)^{2}}\left(q_{j}(k)-\hat{q}_{j}(k)\right)^{2}
$$

where $\left(q_{j}(k), \hat{q}_{j}(k)\right), j=1, \ldots, 2 n$ stand for the current state of the biped and its $\mathrm{NN}$ respectively. $\sigma_{j}(k)$ 
is the standard deviation of each RLC variable $\hat{q}_{j}$ at sample $k$.

\subsection{Interpretation of the algorithm}

The algorithm proposed in this paper provides two output variables that are interesting to evaluate the dynamic stability of a walker. On the one hand, the NNGI relates the actual state of the biped to one state in its average limit cycle, the RLC. On the other hand the D-statistic is a weighed measure of the deviation between them and constitutes a linearized estimate of the basin of attraction. They can be plotted as in Fig. 3. The upper panel shows the NNGI (dashed line) and the RLC (solid line). When the robot is walking stably the NNGI will track the RLC very closely (two first strides in the figure). However, if it suffers a perturbation the NNGI will deviate as happens in the third stride. The lower panel provides the D-statistic (solid line), the deviation measurement. Its interpretation is straightforward: The larger it is, the further the robot is from its limit cycle. Based on a series of stable and perturbed walking experiments the designer can select a threshold for the D-statistic ( $D_{t h}$, dashed line) that establishes whether the robot is about to suffer a fall, or it will continue walking stably, mimicking the concept of the basin of attraction.

\section{Evaluation of the NNGI for detection of perturbations in a Limit Cycle Walker prototype}

This section presents the evaluation of the proposed technique with a Limit Cycle Walker prototype. After an overview of the robot, we summarize how the parameters of the algorithm were selected. At last, experimental results of stable and perturbed walking are provided.

\subsection{System overview of Meta}

Meta is a "four-legged biped" developed at Delft University of Technology [12]. Mechanical coupling between inner and outer leg pairs makes it walk in an almost straight line, i.e. the dynamic behavior of the walker is almost two-dimensional. The prototype has seven body parts (an upper body, two upper legs, two lower legs, and two feet) and the same amount of DoF, located at the body (external DoF), hips, knees, and ankles, Fig. 4a.

Meta has four powered DoF, both ankles and hips, actuated with DC motors placed close to the hip. This configuration keeps the limbs inertia low. Series Elastic Actuation interfaces ankle joints with their actuators [25], reducing the interface stiffness, enhancing shock tolerance and decreasing the amount

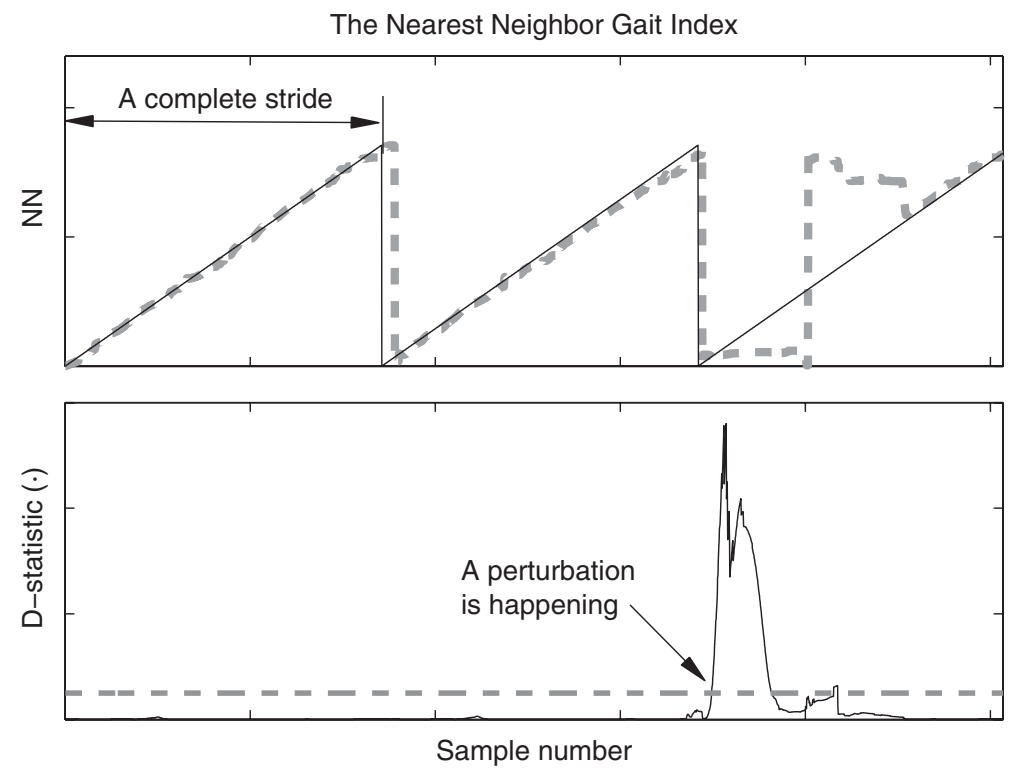

Fig. 3. An example of the execution of the proposed method. The upper panel shows the NNGI (dashed line) and the RLC (solid line). The lower panel shows the D-statistic (solid line) and its threshold $D_{t h}$ (dashed line). 
(a)
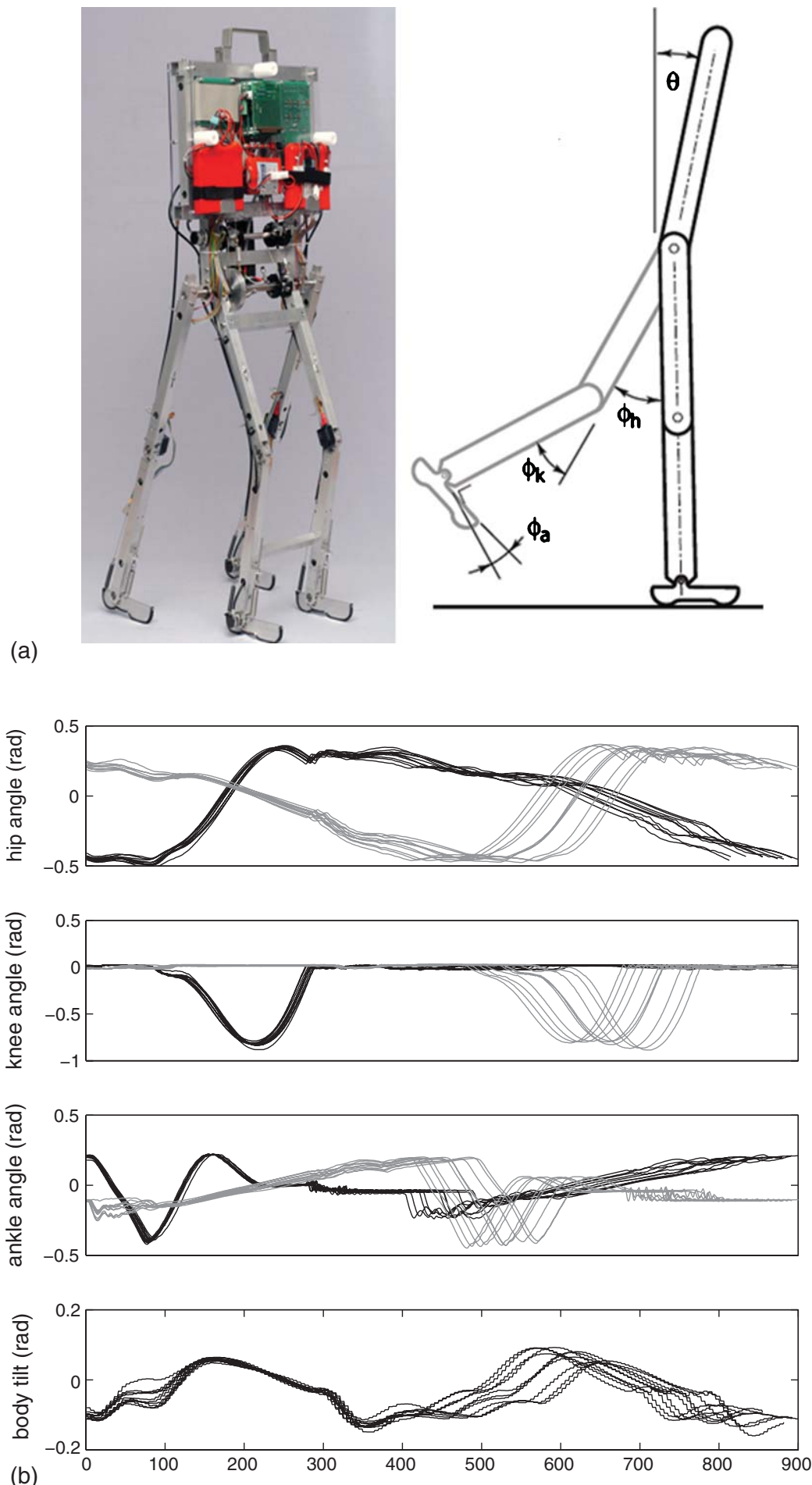

Fig. 4. Limit Cycle Walker Meta: Appearance, schematic representation, and joint angles during normal walking. (a) Appearance and schematic representation depicting the DoF of Meta. Because of the coupling between the inner and outer pairs of legs the walker may be thought of as two dimensional. (b) Joint angles (rad) for the hip, knee, ankle, and body tilt of 10 (out of 28) strides employed to calculate the RLC of Meta. Data sampled at $500 \mathrm{~Hz}$. The black and grey lines represent the inner and outer legs respectively. 
of reflected inertia. Moreover, ankle joints can be torque controlled measuring the elongation of the elastic element. The passive knees are equipped with a solenoid-driven latch which unlocks the knee at the start of the swing phase.

Angles of the six joints are measured with incremental encoders. They provide a resolution of $4 \times 10^{-4} \mathrm{rad}$ for the hip, $3 \times 10^{-4} \mathrm{rad}$ for the knee, and $2 \times 10^{-4} \mathrm{rad}$ for the ankle joints. Body tilt is obtained with a vestibular organ that consists of three accelerometers and three gyroscopes. Ground contact is detected by one switch placed underneath each foot. Sensory data is sampled at $500 \mathrm{~Hz}$ rate. Meta is controlled with a PC/104 stack that includes a $400 \mathrm{MHz}$ processor. The gait controller consists in a state machine that provides both feedback and feedforward commands.

\subsection{Preliminary calculations and selection of parameters}

Limit Cycle Walkers rely on reduced sensory information for gait control. Usually, as in Meta, angular rate is obtained by numeric differentiation of the encoder signals employed to measure joint angles, a procedure that amplifies high frequency noise. Considering that limit cycle walking requires low bandwidth, high frequency noise can be removed by low-pass filtering. To allow for fast detection of perturbations the filter selected must introduce no delay. We have chosen the Benedict-Bordner filter, a tracking algorithm that provides an optimal trade-off between signal smoothing and tracking [4], because achieves good filtering with zero phase.

Before running the NNGI, the normal walking pattern of the robot (RLC) must be obtained. In this case we made Meta walk 14 runs on a carpet, Fig. 4b. Only two of the last strides of each run were considered to avoid transient effects. Signals were processed as described in Section 2.2.1, and next joint velocities were filtered; afterwards the RLC and its standard deviation for a stride of average duration, 1.711s (standard deviation $0.073 \mathrm{~s}$ ), were obtained. The RLC has a length $r=855$ samples at $500 \mathrm{~Hz}$.

As explained above, the perturbation detection method has two parameters that need to be tuned because they depend on the characteristics of the robot. These parameters are:

- The length of the Ensemble of Candidate Neighbors (ECN), $m$. The number of states within the
$\mathrm{ECN}$ is related to the inter-stride variability, and depends on the duration of a complete stride, the sensors sampling rate, and the capability of the onboard computer of the walker. As said in Section 2.1.2, if $m$ is too large the algorithm may ignore the occurrence of a perturbation, while if it is too small it may provide false positives. Figure $5 \mathrm{~b}$ shows an example of the influence of $m$ : If $m \geq 60$ the algorithm fails detecting a (tangential) perturbation that causes a fall; neither the NNGI deviates from the RLC, nor the D-statistic increments its value. On the contrary, if the designer chooses an ECN too short, the algorithm can provide false detections, as it does in Fig. 5a, b when $m=30$. However for $m \geq 40$ it provides the same results, showing that the robot is walking stably. From the execution of the proposed algorithm with all the available trials, we observe that it achieves an optimal performance (no false detections, and a 100\% detections) for a value $m=50$.

- The threshold on the D-statistic, $D_{t h}$. As said in subsection 2.2 this threshold is an experimental approach to being inside or outside the basin of attraction. If the designer chooses a small value for $D_{t h}$, the algorithm will detect perturbations that could be rejected naturally, but if its too large, it will fail to detect that the robot is being perturbed, or it will not provide a sufficiently fast detection. Again it must be tuned after executing a number of trials with and without perturbations. From the experiments presented in this paper, we conclude that a threshold $D_{t h}=100$ avoids false detections because the highest value of $D$ during normal walking is $D=60.80$.

Table 1 summarizes the results for different values of $m$. In the table P stands for a push perturbation and $\mathrm{T}$ for a trip, the number represents the trial. After the tuning process, it is concluded that the value of $D_{t h}$ has little influence in the performance of the algorithm compared with $m$. It only affects the detection delay. Once we have selected a value that avoids false positives during normal walking, the detection delay varies less than $5 \mathrm{~ms}$ if $D \leq 2000$.

\subsection{Stable walking experiments}

First, we executed the algorithm during a series of stable walking trials. As expected, the NNGI tracked 
(a)
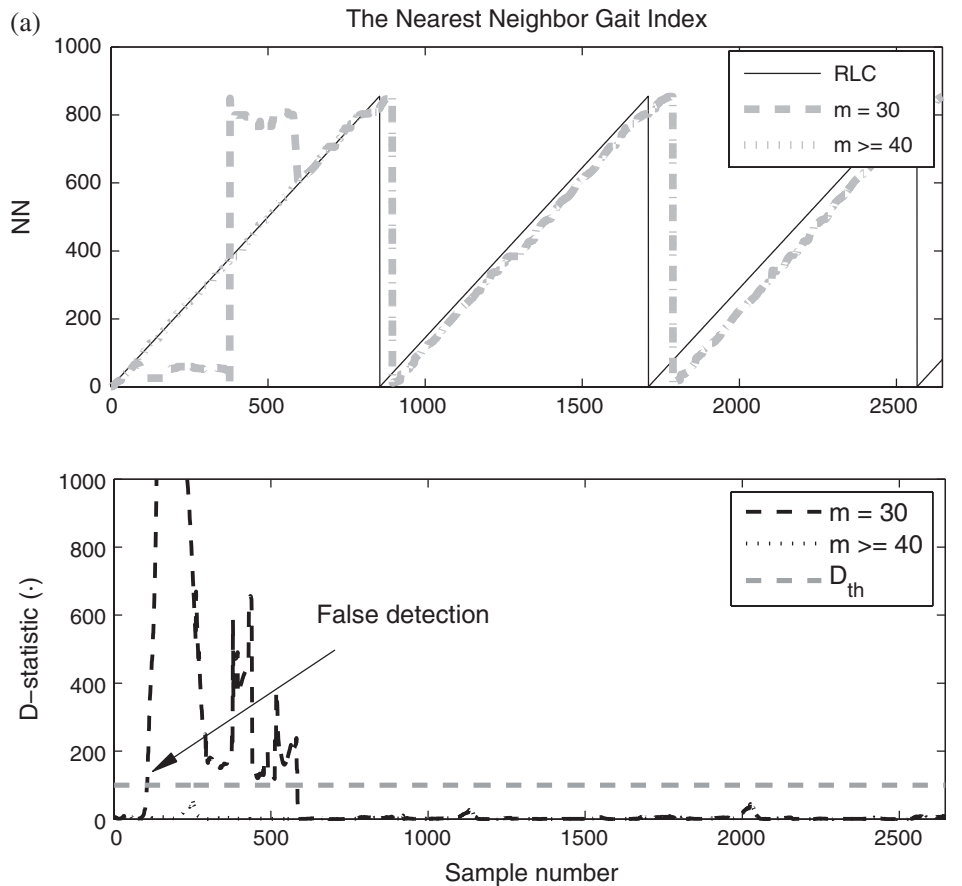

(b)
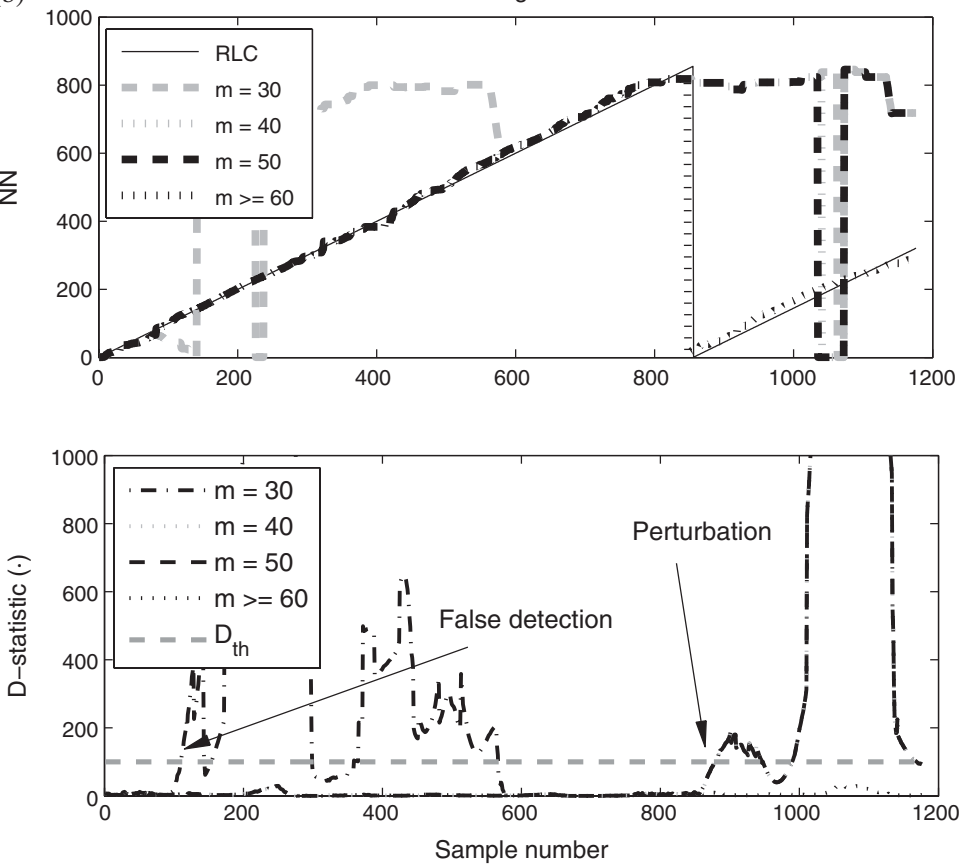

Fig. 5. Selection of the parameters of the algorithm: The length of the Ensemble of Candidate Neighbors, $m$ (top), and the threshold for the D-statistic, $D_{t h}$ (bottom). (a) Execution of the NNGI in one stable run of Meta. Top: The solid black line represents the RLC, the NNGI for different values of $m$ is shown as dashed gray lines. Botton: Dashed and dotted black lines represent different values of $m$, the dashed gray line the D-statistic. (b) Execution of the NNGI during trip trial 1 (which ended in a fall). Top: The solid black line represents the RLC, the NNGI for different values of $m$ is shown as dashed gray and black lines. Bottom: The four black lines represent different values of $m$, the dashed gray line the D-statistic. 
Table 1

Influence of parameter $m$ in perturbation detection performance

\begin{tabular}{lll}
\hline$m$ & False positives & No detection? \\
\hline 30 & Always & Always \\
40 & $\mathrm{P}^{2}, \mathrm{~T}^{2}$ & - \\
50 & - & - \\
60 & - & $\mathrm{P} 3, \mathrm{~T} 1, \mathrm{~T} 3$ \\
70 & - & $\mathrm{P} 1^{1}, \mathrm{P} 3, \mathrm{~T} 1, \mathrm{~T} 3, \mathrm{~T} 4$ \\
80 & - & $\mathrm{P}^{1}, \mathrm{P} 3, \mathrm{P} 4{ }^{1}, \mathrm{~T} 1, \mathrm{~T} 3, \mathrm{~T} 4$ \\
100 & - & $\mathrm{P}^{1}, \mathrm{P} 3, \mathrm{P} 4{ }^{1}, \mathrm{~T} 1, \mathrm{~T} 3, \mathrm{~T} 4$ \\
\hline
\end{tabular}

${ }^{1}$ During the perturbation the $\mathrm{D}$-statistic reaches a peak value not much larger than in normal walking, e.g. below 200. This threatens successful detection.

2 The robot suffered a perturbation but did not deviate from its limit cycle, which indicates that it kept inside the basin of attraction.

considerably close the RLC, but with subtle differences in joint trajectories, velocities, and stride duration. Figure 6 shows an example of how the NNGI and the D-statistic evolve during three stable strides. It is observed that the NNGI tracks closely the RLC, with only negligible deviations, indicating that the biped is following its RLC. Concerning to the D-statistic, it presents peaks of 56.04, 31.34, and 47.74 around samples number 250, 1100, and 2000 respectively. This corresponds to the heel strike of the outer legs. Table 2 depicts the peak values of the D-statistic for 10 stable strides. All of them appear also when the outer legs impact the ground. The reason for this is two fold: First, the synchronization of the outer legs happens just before heel strike (originating the most considerable inter-stride variation), and second, because of an insufficient sampling frequency of the RLC, which does not let the algorithm record a state more similar (closer) to the current one. Nevertheless, increasing the amount of points of the RLC would require an excessive amount of memory and computational resources of the onboard computer; as said, it is more effective to ignore these peaks selecting a value for the D-statistic threshold $D_{t h}=100$.

Figure 7 shows the calculation of the NN during the whole experiment. It represents the Euclidean distance at every point during the walk. As the prototype is walking stably the NN follows the RLC, which implies that the point with least distance to the current state of the walker (solid black line) is always in the neighborhood of the expected state, i.e. candidate number 0 ; thus the robot is walking in a limit cycle.

\subsection{Perturbed walking experiments}

Together with the stable walking experiments, a series of trials where the walker suffered a push or a trip were performed. They served to validate the pro-
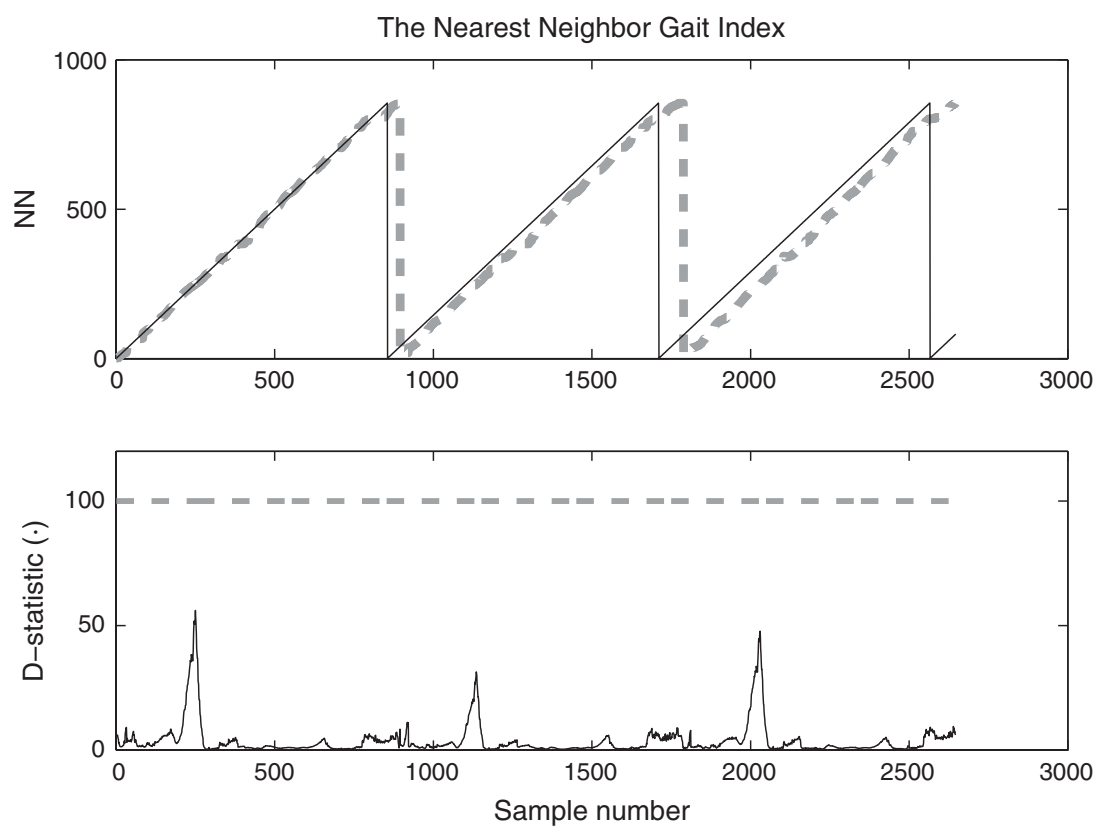

Fig. 6. Upper panel: the dotted line represents the NN for three consecutive stable strides as provided by the NNGI. The solid line corresponds to the RLC, the dashed line to the NNGI. The lower panel shows the D-statistic (solid line) and the selected threshold $D_{t h}$ (dashed line). 
Table 2

D-statistic for 10 stable walking strides

\begin{tabular}{lccccc}
\hline Stride & 1 & 2 & 3 & 4 & 5 \\
Max (D-st) & 56.04 & 31.34 & 47.74 & 30.04 & 36.96 \\
\hline Stride & 6 & 7 & 8 & 9 & 10 \\
Max (D-st) & 60.80 & 41.07 & 16.09 & 49.76 & 30.64 \\
\hline
\end{tabular}

posed technique and, as explained before, to select the adequate value for the parameters. Trip experiments consisted in an obstacle made of steel placed on the path of the robot, so that the biped stumbled with it at different gait phases. Pushes where gently applied by an experimenter at the body of the robot, also at different moments in the gait cycle. Figure 8 shows the execution during a push experiment. As a result of the disturbance, the walker deviates dramatically from its RLC, as it can be observed in the upper plot; moreover, the D-statistic reaches to a high value, 5600.5, about one hundred times larger than in stable walking. In spite of this, the biped is able to recover from the perturbation by putting its swinging leg quickly on the floor, performing a so-called "lowering strategy" in humans [9]. Table 3 summarizes the peak values of the D-statistic during four trip and push experiments. The D-statistic overpasses the selected threshold $\left(D_{t h}=100\right)$ for all the experiments in which the robot falls, but it provides three "false alarms" when the robot suffers a push (experiments number 1, 2, and 4). In two of these cases (experiments 1 and 4) the robot almost fails to perform the subsequent ankle push off, which is the major cause of falling in the ensemble of push and trip experiments; therefore it would be preferable to trigger a recovery reaction to avoid a potential fall. This is shown in Figure 9a where we observe that the ankle angle of the inner leg at push-off after the perturbation is abnormally small (for experiment 1). Experiment 2, on the other hand, exhibits no noticeable differences in leg angles with respect to unperturbed trials, only decreased forward tilt in the stride after the perturbation, Figure $9 \mathrm{~b}$. Since joint angles (the other state variables) follow normal profiles, the D-statistic keeps low values, not indicating the occurrence of the perturbation. Related to experiment 3 , the peak in the D-statistic happens 8 milliseconds after the heel strike of the outer legs, thus it could be related to the small peaks that happen at that moment in normal walking but amplified by the push.

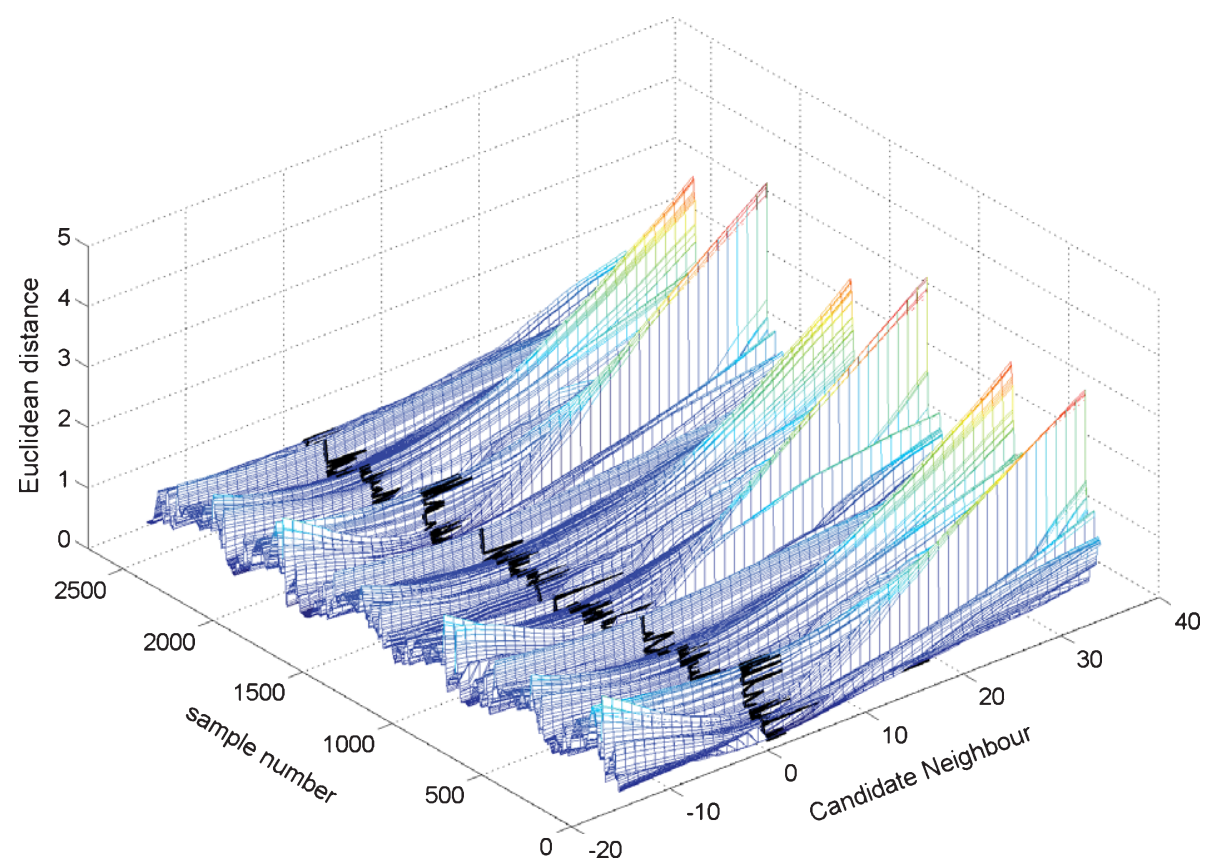

Fig. 7. Calculation of the NN during a stable walking experiment. The surface represents the Euclidean distance to every candidate neighbour at every sample of the trial, the black solid line the NN. 

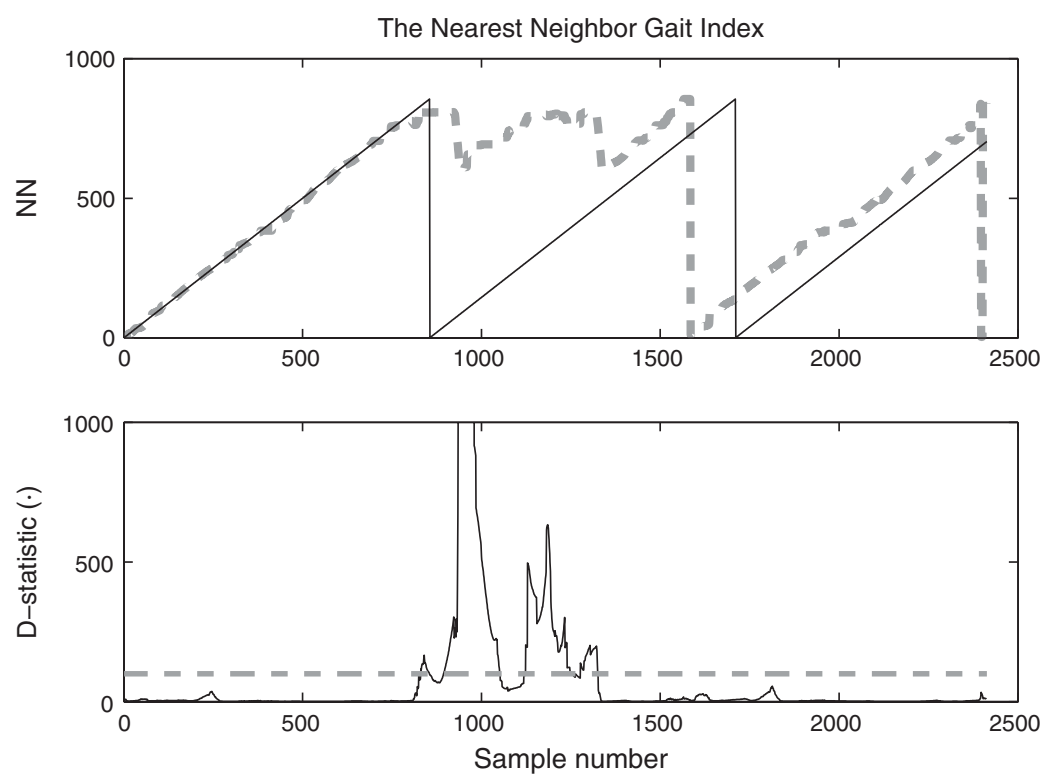

Fig. 8. Upper panel: the dahsed line represents the NN for push trial 3 (the robot kept walking) as provided by the NNGI. The solid line corresponds to the RLC, the dashed line to the NNGI. The lower panel shows the D-statistic (solid line) and the selected threshold $D_{t h}$ (dashed line).

Table 3

D-statistic for 8 perturbed experiments

\begin{tabular}{lcccc}
\hline Push trial & 1 & 2 & 3 & 4 \\
Max(D-st) & 5600.5 & 63.17 & 4967.8 & 6227.4 \\
Fall? & $\mathrm{No}^{1}$ & No & No & $\mathrm{No}^{1}$ \\
\hline Trip trial & 1 & 2 & 3 & 4 \\
Max(D-st) & 18074 & 54.40 & 2523.3 & 6487.0 \\
Fall? & Yes $^{2}$ & No & Yes $^{2}$ & Yes $^{3}$ \\
\hline
\end{tabular}

1 The robot experiences notable problems to perform ankle push off after the perturbation, but it succeeds.

2 The fall is caused because the heel strike after the perturbation is not performed with the legs completely outstretched.

3 Ankle push off after the perturbation is not executed adequately; the walker falls backwards.

\section{Discussion}

This paper focuses on a new method to monitor the stability of a Limit Cycle Walker based on a normal walker pattern (the RLC) and a static measurement of dynamic stability (the D-statistic). It can be applied not only to detect gait perturbations, but also to quantify dynamic stability. A brief discussion on these topics is provided next.

\subsection{Application to perturbation detection}

The experimental results presented in Section 3 indicate that the implementation of the proposed algorithm provides a technique to detect the occurrence of an unexpected event that may lead the robot to a fall. In fact, for the selected set of parameters, the algorithm provides no false detections when the robot is walking stably, and also has a $100 \%$ success rate detecting perturbations that cause a fall. Three pushes (push experiments 1, 3 and 4) that did not make the robot collapse where identified as perturbations, but it was observed that the robot experienced serious difficulties avoiding a backwards fall in the subsequent stride in experiments 1 and 4 and that in experiment 3 the "false alarm" happened because the push amplified the variations that always happen at heel strike of the outer legs.

Related to the delay in perturbation detection, it must be pointed out that in the current setup the occurrence of the perturbation was recorded with a conventional camera that provides $30 \mathrm{~Hz}$ sampling rate. Thus, the moment at which the perturbation happens can be estimated with roughly $17 \mathrm{~ms}$ error. This error was partially compensated assuming that the perturbation happens when the monotonic increase in the D-statistic (just before when the perturbation is detected) begins. The average delay was estimated as $45.2 \mathrm{~ms}$ (standard deviation $3 \mathrm{~ms}$ ), therefore comparable to short latency reflexes in humans, typically estimated to be around $35 \mathrm{~ms}$ [30] Note that humans serve as reference in biomimetic gait control research. Table 1 summarizes 

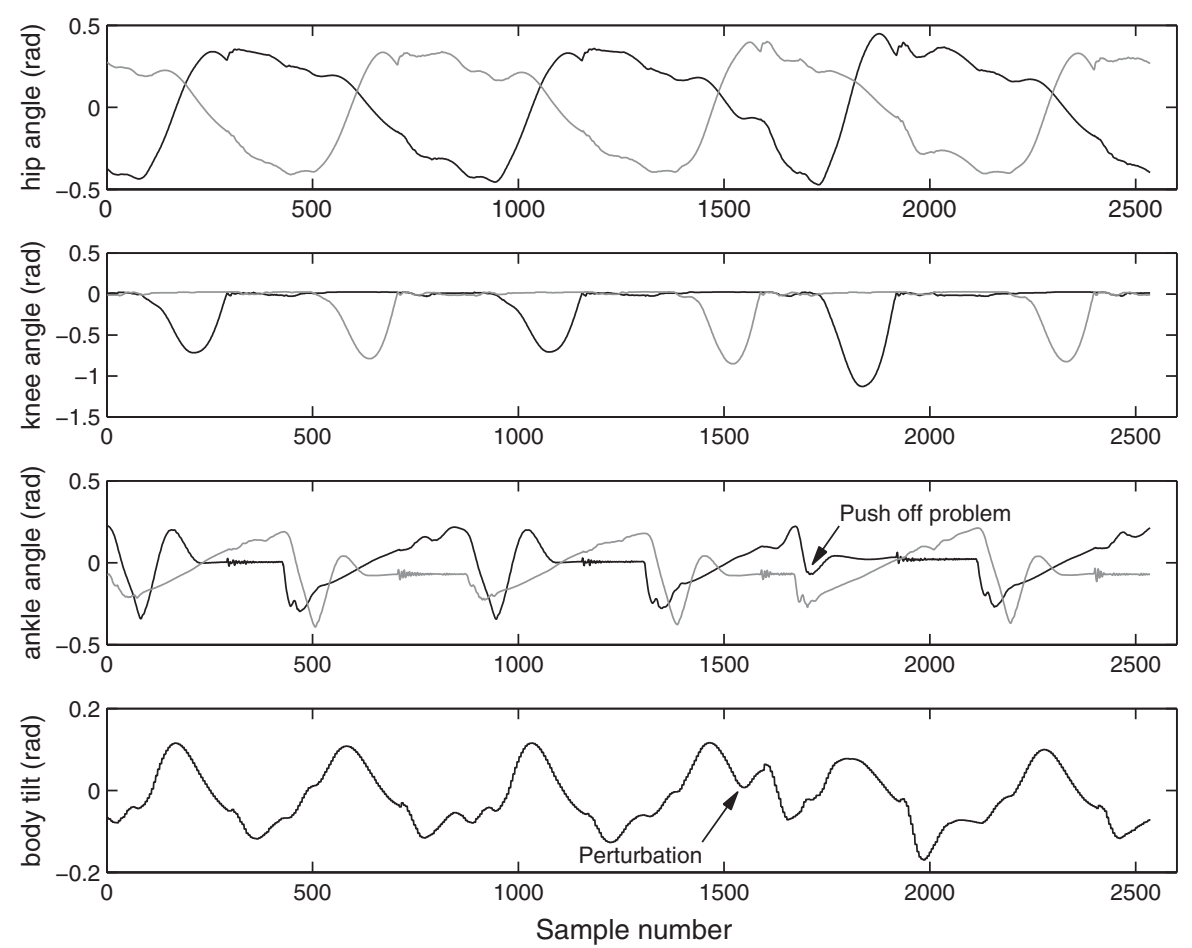

(a) Joint angles during push trial 1 .
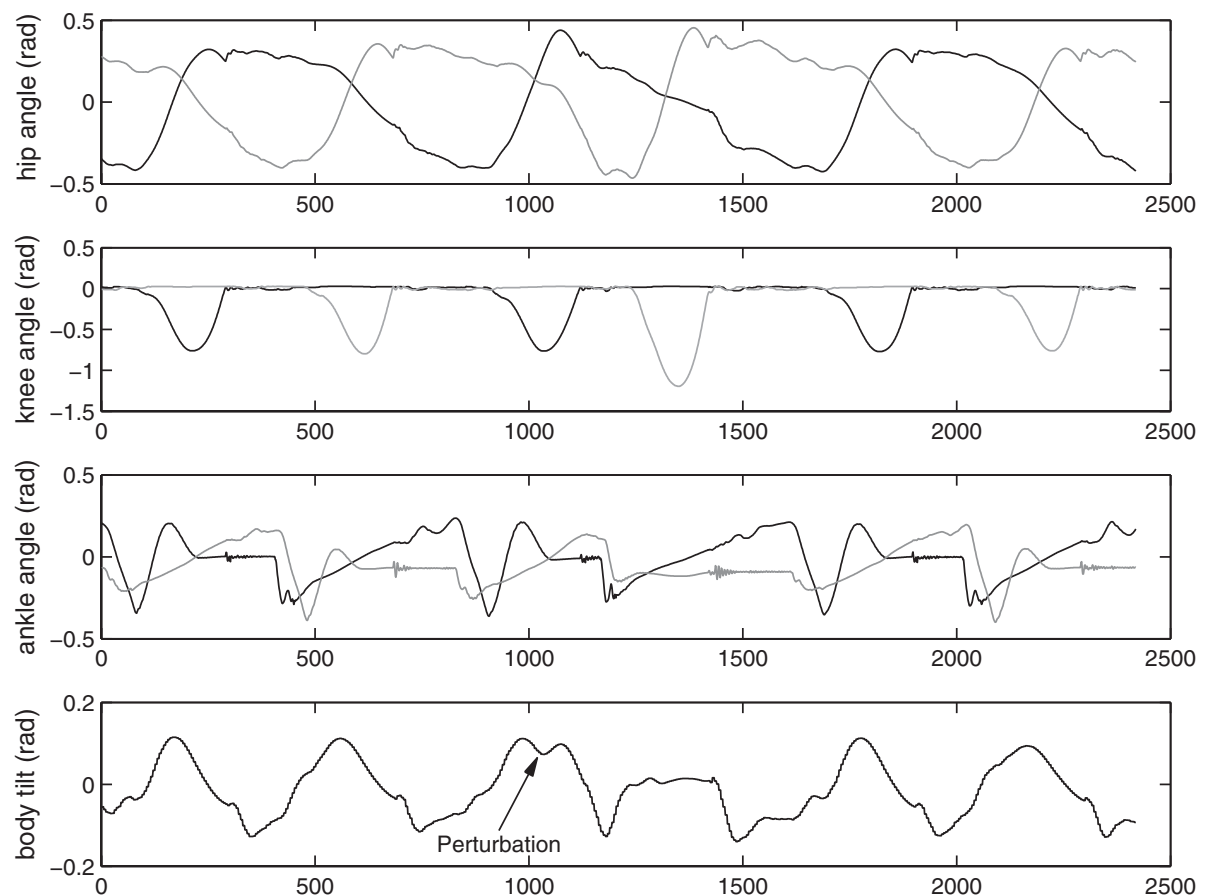

(b) Joint angles during push trial 2.

Fig. 9. Joint angles (rad) for the hip, knee, ankle, and body tilt during push trials 1 and 2. Data sampled at $500 \mathrm{~Hz}$. The black and grey lines represent the inner and outer legs respectively. The moment at which the perturbation is applied is indicated by an arrow in the body tilt plot. 
the performance in perturbation detection based on different values of $m$. The second parameter, $D_{t h}$, only affects in detection time in always less than $5 \mathrm{~ms}$ above the value that avoids false positives during normal walking (60.80), and below 2000. Thus it is not thoroughly described. Current work on the implementation of the NNGI in a new bipedal robot and its simulation model will provide a more precise measure of the detection delay and the influence of the parameters $D_{t h}$ and $m$ in the final results.

Moreover, we are working on the validation of our perturbation detection algorithm in the novel ESBiRRo exoskeleton, a hip-knee-ankle-foot orthosis (HKAFO) based on the concept of Limit Cycle Walking, Fig. 10. The ESBiRRo exoskeleton consists of variable stiffness ankle and knee joints and active hip joints, driven by flat DC motors. Like this, the exoskeleton has most of the weight distributed proximally, not influencing the subjects dynamics, and letting the human-robot system settle naturally into a Limit Cycle.

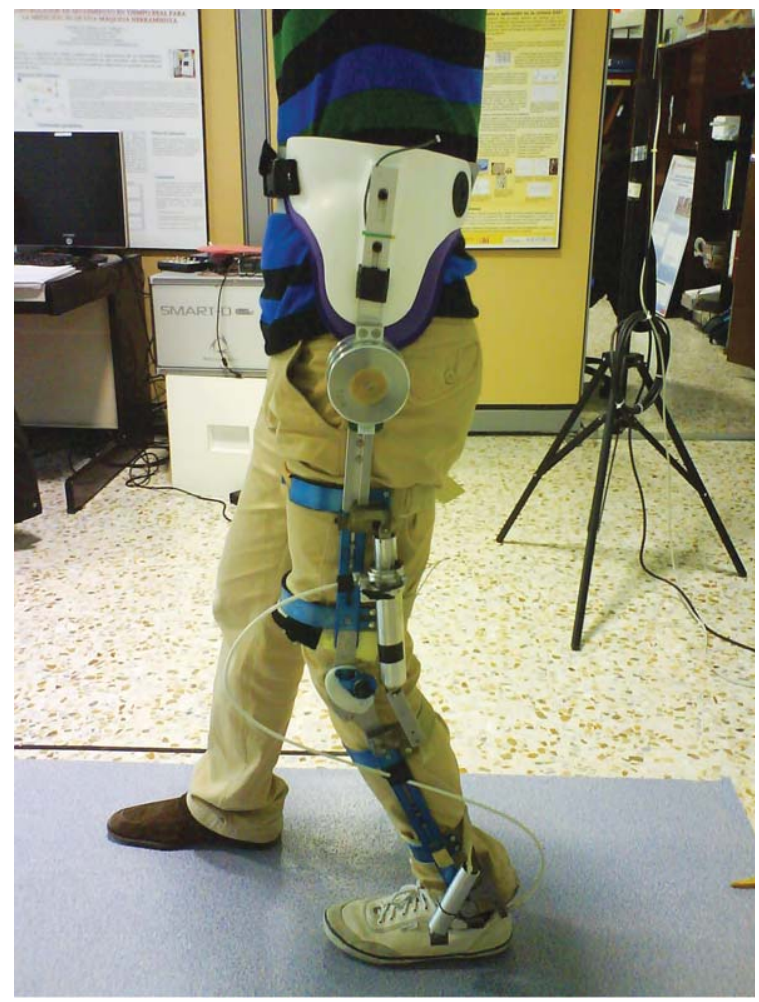

Fig. 10. Lateral view of ESBiRRo HKAFO exoskeleton depicting DC flat motors at the hip, and variable stiffness actuators at knee and ankle.

\subsection{A novel tool to assess dynamic stability}

Although this paper focuses on perturbation detection, the core of the proposed method is to provide a linearized measurement of the dynamic stability of a walker. As reviewed in the introduction, the stability of Limit Cycle Walkers has been traditionally assessed in a step-to-step basis, i.e. slicing the limit cycle at one fixed point (for example at heel strike of a given leg) and calculating the inter-stride variation in this fixed Poincaré section. The way to measure this variation changes with the technique; it can be based, for example, on the calculation of Floquet Multipliers [15], or on a series of gait indicators [11]. However, these methods ignore what is happening in all the other states of the gait cycle.

The application of the proposed method to a walking prototype yielded some insight into its inherent dynamic stability. Notice that the dynamic stability of the walker is influenced not only by its walking controller, but also by its mechanical design. Our major discoveries in Meta were: 1) during unperturbed walking, the largest inter-stride variation happened at heel strike of the outer legs because of their synchronization mechanism, 2) perturbations at mid and late swing cause a "fast advance" in the tangential direction of the limit cycle (a so-called lowering strategy in humans [9]), but the robot does not fall if it can perform a sufficiently powerful ankle push off afterwards (this agrees with previous experiences demonstrating the role of ankle push off in walking stability and energetics [13, 19]), and 3) perturbations at early swing make the robot fall because the swinging legs land not completely stretched, making the knees collapse. These results suggest that the application of the NNGI method may help us to understand how different factors affect the dynamic stability of a given robot. Moreover, with help of an adequate benchmark, the NNGI could be used to compare the stability of different walking machines or control techniques, measuring how the same perturbation affects them.

\section{Conclusions}

This paper presented a method for detection of gait perturbations in bipedal walkers, both humanoid robots and exoskeletons. The algorithm monitors online the state of the robot and decides whether a perturbation is happening based on a phase representation of the nor- 
mal walking of the biped. It presents the advantages of having low computational cost and avoiding the need of a model of the robot dynamics, just a reference gait pattern. Moreover, the algorithm has only two parameters the designer needs to tune; among them only one has a large influence in the performance of the algorithm, thus it is quickly to adjust. This is done with data from a reduced number of stable and perturbed walking trials. The proposed method was validated with a Limit Cycle Walker prototype providing a $100 \%$ success rate in perturbation detection for the current experimental apparatus and adequately tuned parameters, with no false positives when the robot is walking unperturbed. The average delay in disturbance detection is $45 \mathrm{~ms}$, comparable to short latency reflexes in humans.

\section{Acknowledgement}

The work presented in this paper has been carried out with the financial support from the Commission of the European Union, within Framework 6, ICT program "Cognitive Systems, Interaction, Robotics," Key Action 2.6.1 "Advanced Robotics," under contract IST-61-045301-STP, "ESBIRRO - Biomimetic actuation, sensing and control technology for limit cycle bipedal walkers," and from the Ministerio de Ciencia e Innovación, Programa CONSOLIDER - INGENIO, under contract "HYPER - Hybrid neuroprosthetic and neurorobotic devices for functional compensation and rehabilitation of motor disorders."

The authors would like to thank to Martijn Wisse, Daan G.E. Hobbelen, and J.G. Daniël Karssen, from Delft U.T., and Eduardo Rocon and Alfonso Montellano from CSIC, for their help with the experiments.

\section{References}

[1] A.A. Ahmed and J.A. Ashton-Miller, On use of a nominal internal model to detect a loss of balance in a maximal forward reach, J Neurophysiol 97(3) (2007), 2439-2447.

[2] F. Ali and M. Menzinger, On the local stability of limit cycles, Chaos 9(2) (1999), 348-356.

[3] S. Au and H. Herr, Powered ankle-foot prosthesis, IEEE Robotics \& Automation Magazine 15(3) (2008), 52-59.

[4] T.R. Benedict and G.W. Bordner, Synthesis of an optimal track-while-scan smoothing equations, IRE Trans Automat Control 7(4) (1962), 27-32.

[5] S.J. Blakemore, C.D. Frith and D.M. Wolpert, The cerebellum is involved in predicting the sensory consequences of action Neuroreport 12(9) (2001), 1879-1884.
[6] D.J. Braun and M. Goldfarb, A control approach for actuated dynamic walking in biped robots, IEEE Transactions on Robotics 25(6) (2009), 1292-1303.

[7] J. Duysens and H.W. van de Crommert, Neural control of locomotion; The central pattern generator from cats to humans, Gait Posture 7(2) (1998), 131-141.

[8] J.J. Eng, D.A. Winter and A.E. Patla, Strategies for recovery from a trip in early and late swing during human walking, Exp Brain Res 102(2) (1994), 339-349.

[9] A. Forner-Cordero, H.F.J.M. Koopman and F.C.T. van derHelm, Multiple-step strategies to recover from stumbling perturbations, Gait Posture 18(1) (2003), 47-59.

[10] A. Forner-Cordero, H.J.F.M. Koopman and F.C.T. van derHelm, Describing gait as a sequence of states, $J$ Biomech 39(5) (2006), 948-957.

[11] D.G.E. Hobbelen and M. Wisse, "Limit Cycle Walking", Humanoid Robots, Human-Like Machines. I-Tech Education and Publishing, 2007, pp. 277-294.

[12] D.G.E. Hobbelen and M. Wisse, A disturbance rejection measure for Limit Cycle Walkers: the gait sensitivity norm 23(6) (2007), 1213-1224.

[13] D.G.E. Hobbelen and M. Wisse, Ankle actuation for Limit Cycle Walkers, The International Journal of Robotics Research 27(6) (2008), 709-735.

[14] O. Höhn and W. Gerth, Probabilistic balance monitoring for bipedal robots, Int J Rob Res 28(2) (2009), 245-256.

[15] Y. Hurmuzlu, Dynamics of bipedal gait; Part II: Stability analysis of a planar five-link biped, ASME Journal of Applied Mechanics 60 (1993), 337-343.

[16] T. Ishida, Development of a small biped entertainment robot QRIO In: Proc International Symposium on MicroNanomechatronics and Human Science and the Fourth Symposium Micro-Nanomechatronics for Information-Based Society, 2004, pp. 23-28.

[17] J.G.D. Karssen and M. Wisse, Fall detection in walking robots by multi-way principal component analysis, Robotica 27(2) (2008), 249-257.

[18] M. Kawato, Internal models for motor control and trajectory planning, Current Opinion in Neurobiology 9 (1999), 718727.

[19] A.D. Kuo, Energetics of actively powered locomotion using the simplest walking model, J Biomech Eng 124(1) (2002), 113-120.

[20] R.R. Llinás, I of the vortex: From neurons to self, MIT Press, 2001.

[21] M. Nakanishi, T. Nomura and S. Sato, Stumbling with optimal phase reset during gait can prevent a humanoid from falling, Biol Cybern 95(5) (2006), 503-515.

[22] K. Ogata, K. Terada and Y. Kuniyoshi, Falling motion control for humanoid robots while walking In: IEEE-RAS 7th International Conference on Humanoid Robots, 2007.

[23] J.L. Pons, ed., Wearable Robots: Biomechatronic Exoskeletons, John Wiley \& Sons, Ltd, 2008.

[24] V. Prahlad, D. Goswami and C. Meng-Hwee, Disturbance rejection by online ZMP compensation, Robotica 26(1) (2008), 9-17.

[25] G.A. Pratt and M.M. Williamson, Series elastic actuatorsIn: IEEE/RSJ International Conference on Intelligent Robots and Systems, vol. 1, 1995, pp. 399-406.

[26] R. Renner and S. Behnke, Instability detection and fall avoidance for a humanoid using attitude sensors and reflexes In: Proc IEEE/RSJ International Conference on Intelligent Robots and Systems, 9-15 Oct., 2006, pp. 2967-2973. 
[27] Y. Sakagami, R. Watanabe, C. Aoyama, S. Matsunaga, N. Higaki and K. Fujimura, The intelligent ASIMO: system overview and integration In: IEEE/RSJ International Conference on Intelligent Robots and System, Vol. 3, 2002, pp. 2478-2483.

[28] A.M. Schillings, B.M. van Wezel, T. Mulder and J. Duysens, Muscular responses and movement strategies during stumbling over obstacles, J Neurophysiol 83(4) (2000), 2093-2102.

[29] S. Strogatz, Nonlinear Dynamics and Chaos, Cambridge, Westview Press, MA, USA, 2000.
[30] M.H. van der Linden, D.S. Marigold, F.J.M. Gabre 'ls and J Duysens, Muscle reflexes and synergies triggered by an unexpected support surface height during walking, J Neurophysiol 97(5) (2007), 3639-3650.

[31] E.R. Westervelt, J.W. Grizzle and D.E. Koditschek, Hybrid zero dynamics of planar biped walkers, IEEE Transactions on Automatic Control 48(1) (2003), 42-56.

[32] D.M. Wolpert, R.C. Miall and M. Kawato, Internal models in the cerebellum, Trends in Cognitive Sciences 2(9) (1998), 338-347. 

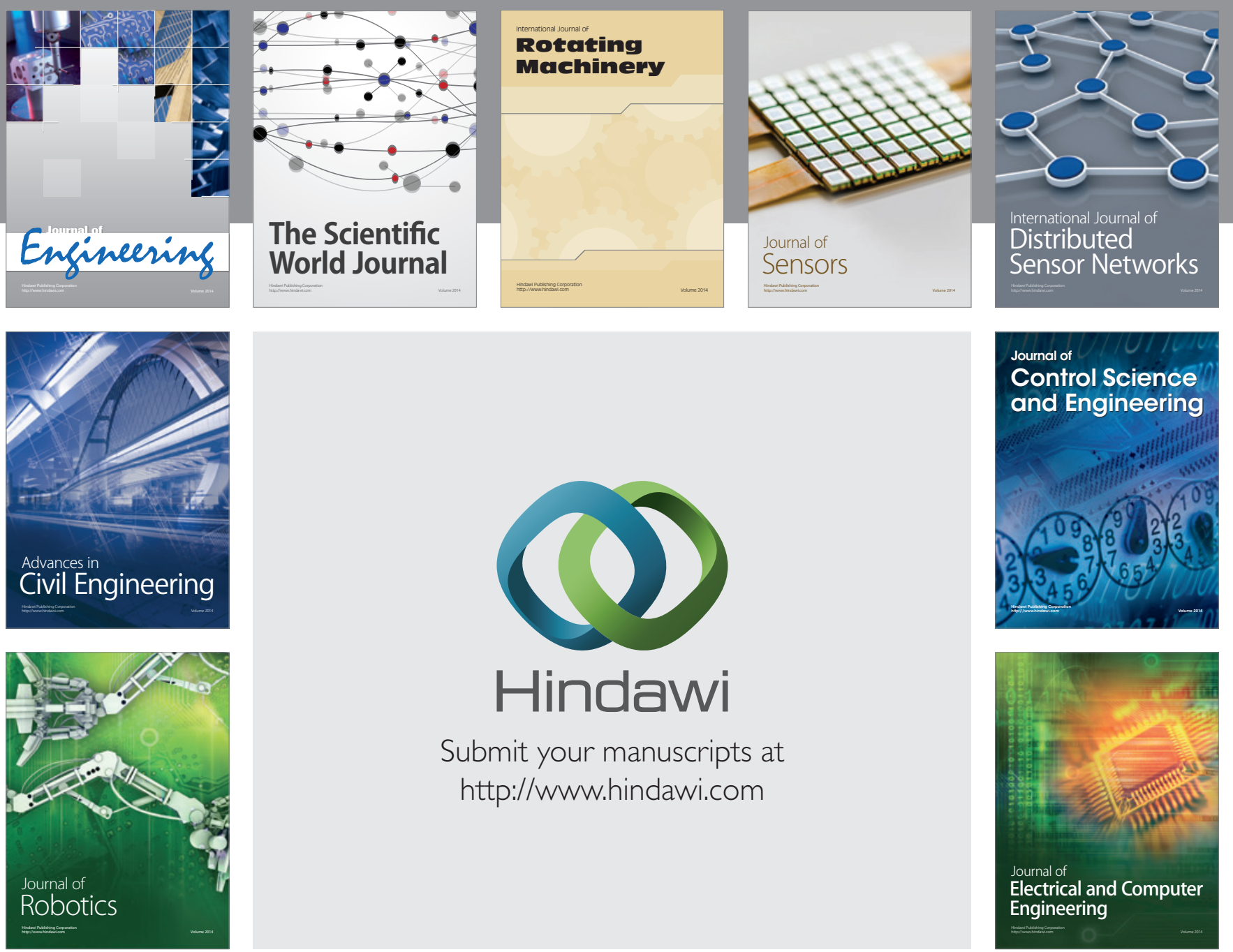

Submit your manuscripts at

http://www.hindawi.com
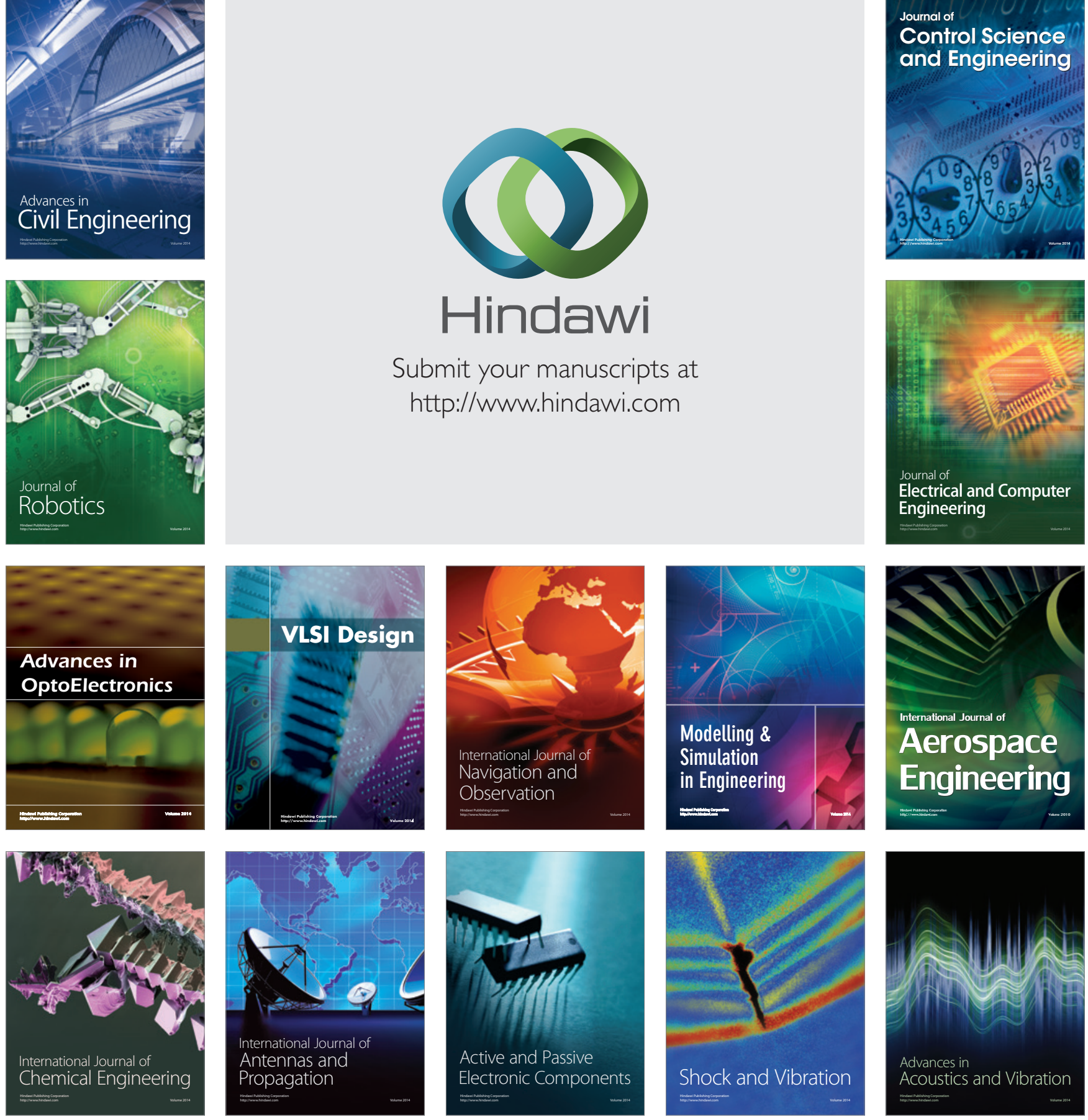